In der administrativen Sitzung vom 30. 6. 1951 äußerte die zur Jahresversammlung in Vevey vereinte Gesellschaft den Wunsch, es möchte der diesjährige Sitzungsbericht von einem Rückblick auf die ersten 10 Jahre des Gesellschaftslebens begleitet sein, und bestimmte auf Antrag des Vorstandes A. Gruá́ach zum Referenten. Wenn ich diesen ehrenvollen Auftrag annahm, so ge-schah dies nicht nur, weil ich am Zustandekommen der Gesellschaft führenden Anteil hatte, sondern vor allem, weil ich - wie bereits heute nurmehr wenige - liber thre Entstehung orientiert bin.

In Tat und Wahrheit wurde die Schweizerische Mikrobiologische Gesellschaft zweimal geboren: 1930 in Paris als Société Suisse de Microbiologie, das zweitemal 1942 unter dem ihr heute eigenen Doppelnamen. Erfolgte die erste Gründung in einer relativ fried-lichen Epoche inmitten des glanzvollen Gepräges von Paris, das die Organisation des «Premier Congrès International de Microbiologie» übernommen und unter das Ehrenpräsidium des damaligen Direktors des Institut Pasteur, E. Roux, und das Präsidium von P. Bordet, Direktor des Institut Pasteur von Bruxelles, gestellt hatte, so erfolgte die Auferstehung in einer der weltpolitisch düster-sten und für die Schweiz kritischsten Periode in einem Sitzungs-zimmer des Bahnhofbuffets Zurich.

Th. Madsen, dessen Klugheit und gesellschaftliche Gewandtheit nicht so bald ihresgleichen finden werden, fiel die Ehre zu, nach der Eröffnungsrede Bordets für die ausländischen Delegierten zu sprechen: «Je dois, sans doute, cet honneur au fait que Гidée de la creation d'une Société Internationale de Microbiologie dont émane notre Congrès, a été formulée au cours d'une conference Internationale, la conference de la rage, organisée en 1927 sur Tinvitation de $\Gamma$ lnstitut Pasteur par Torganisation d'Hygiène de la Société des Nations», womit bei der Stellung, die Madsen in alien Komitees der Hygiene-Sektion einnahm, seine persönliche

Pathologie und Bakteriologie, Vol. XIV, Fasc. 5 (1951)

41

63810 Jahre Schweizerische Mikrobiologische Gesellschaft

Bedeutung fur die Gründung der Intemationalen Mikrobiologi-schen Gesellschaft umrissen ist. Ihre hauptsächliche Aufgabe sollte nach Bordet die periodische Organisation von Kongressen sein. Silberschmidt, unter den 5 Lehrstuhlinhabern für Hygiene und damit eo ipso für die medizinische Bakteriologie der einzige Schweizer und auch einzige «Pasteurien», hatte die Schweiz offi-ziell zu vertreten. Wie die große Mehrzahl der Delegierten hatte auch er seine Adresse schriftlich eingereicht. Ich halte daraus fest: «.,.il (le depute de la Suisse) espère que le Comité international voudra bien choisir un de nos centres universitaires suisses comme siege d'une de nos prochaines reunions ....», ein Wunsch, der - wie noch zu zeigen sein wird - durch die Gunst der Ver-hältnisse alle Aussichten auf Erfüllung gehabt hätte, wenn, ja wenn ...

Da dieser Aufsatz von Geschichte handelt, mag es angesichts der Evolution, welche die Mikrobiologie in den seither verflosse-nen 20 Jahren durchlaufen hat, angezeigt sein, zwei Sätze der Ansprache William H. Parks, der für die Delegierten Amerikas sprach, zu zitieren: "We Americans have in the past drawn our new information largely from the researches of European 
workers. Now that we also contribute to the world's store of knowledge, we still look much to the investigators in Europe for the enrichment of our understanding.» Ich darf hoffen, daß dieser Aufsatz auch vom einen oder andern der jetzigen Generation amerikanischer Mikrobiologen gelesen wird. Das mag ihn daran erinnern, daß Amerika die Führung, die es z. Zt. unbestrittenermaßen einnimmt, vielleicht einmal auch wieder abgeben muß - und unsere Zeit schreitet rasch vorwärts. Wie sympathisch, wenn es für unser Empfinden schon heute etwas mehr Verständnis auf-bringen könnte!

Gemäß § 1 der am 25.6. 1930 durch die Generalversamm-lung genehmigten Statuten bilden die Mitglieder der Intemationalen Gesellschaft in ihren eigenen Ländern entweder ein natio-nales Komitee oder nationale Sektionen, die ihrerseits ein natio-nales Komitee wählen.

Die Schweiz hatte denn auch ein nationales Komitee geschaf-fen, bestehend aus den Inhabern der 5 Lehrstühle für Hygiene, d. h.: Cristíani (Genf), Doerr (Basel), Galli-Vallério (Lausanne), Silberschmidt (Zurich), Sobernheim (Bern) und W. Freí von der Veterinär-medizinischen Fakultät Zurich. Es gelang mir nicht, in

10 Jahre Schweizerische Mikrobiologische Gesellschaft

689

Erfahrung zu bringen, ob diese Gesellschaft je konstituiert wurde. Ich hatte stets den Eindruck, daß ich am ersten Kongreß in Paris mit der Aufnahme in die «Internationale» auch Mitglied der «Na-tionalen» geworden sei. In der Folge habe ich aber nie etwas von einer schweizerischen Sektion gehört. Es blieb wohl beim nationalen Komitee, dessen Angelegenheiten dank der freund-schaftlichen Beziehungen, die Silberschmídt mit dem damaligen Direktor des Eidg. Gesundheitsamtes, Dr. Carrière, unterhielt, unter der Hand erledigt wurden. Tatsächlich war 1938 am Zweiten Internationalen Kongreß in London - der 1933 für Berlin vor-gesehene mußte «wegen der Ungunst der Zeit» ausfalien - Sobern-heim der Delegierte der Schweiz. Zum 3. Kongreß nach New York (1939) wurden vom Bundesrat R. Doerr und J. G.Baer delegiert.

In der Liste der in London geschaffenen nationalen Kom-missionen figuriert auch die Schweiz. «The National groups have selected members to represent them on the Permanent International Commission for the Organisation of Congresses.» Die sogenann-le «Schweizerische Mikrobiologen-Gesellschaft» hatte ihr repräsen-tativstes Mitglied $R$. Doerr in dieses Komitee delegiert. Zusammen mit W. Frei vertrat er die «Gesellschaft» auch im Nomenklatur-komitee, ohne daß die Herren m. W. auch nur einer Sitzung bei-gewohnt hätten.

Diese «vie clandestine» der sogenannten Schweizerischen Sektion der «International Association of Microbiologists», wie sie in Abänderung der Pariser Statuten seit dem Londoner Kongreß trotz immer wieder erfolgter Gegenvorschläge - bis heute heißt, war recht eigentlich statutenwidrig und mußte früher oder später zu einer Umgestaltung führen.

Der Vorstoß erfolgte denn auch fast zu gleicher Zeit von zwei ganz verschiedenen Seiten aus einer, ich möchte sagen, «aka-demischen» und einer «nationalen». Der erstere ging von P. Hauduroy (Lausanne) aus, der in durchaus verständlicher Unkennt-nis der bereits bestehenden «Gesellschaft» in einem initiativen Brief die verschiedenen Lehrstuhlinhaber und einige weitere Do-zenten zur Gründung einer schweizerischen Sektion der Internationalen MikrobiologenGesellschaft aufgefordert hat.

Der «nationale» Vorstoß war von weniger emphatischen, da-für aber kräftigeren Worten getragen. Mit Rücksicht auf die in-ternationale Lage hatte ich vom Herrn Oberfeldarzt, Oberstbriga-dier $\boldsymbol{P}$. Vollenweider, den Auftrag erhalten, am Hygiene-Institut

$44^{*}$
640

10 Jahre Schweizerische Mikrobiologische Gesellschaft

der Universität Zurich einen Kurs für Militärbakteriologen zu or-ganisieren. Diese $\Gamma$ Kurs war dringend notwendig geworden, be-saßen wir doch zu jenem Zeitpunkt viel zu wenig in medizinisch-bakteriologischer Diagnostik ausgebildete Armeeangehörige und praktisch 
überhaupt kein armee-eigenes Labormaterial. Bei der Organisation handelte es sieh darum, recht heterogen vorgebildete und den verschiedensten militärischen Graden angehörige Leute auf eine unite de doctrine zu bringen.

Das Ziel des in der Zeit vom 2.-26. 3. 1942 durchgeführten Kurses wurde, wie ich in meinem Bericht an den Herrn Oberfeld-arzt melden konnte, weitgehend erreicht. Darüber hinaus aber entwickelte diese militärische Arbeitsgemeinschaft trotz ihrer zeit-lichen Beschränktheit unter den Teilnehmern ein Zusammenge-hörigkeitsgefühl, aus dem sich weit in die Nachkriegsjahre rei-chende Freundschaften entwickelt hatten. Dem in diesem Kurs unter dem Druck der äußern Ereignisse entstandenen Korpsgeist verdankt die Schweizerische Mikrobiologische Gesellschaft ihre Geburt. Die Kursteilnehmer empfanden das Bedürfnis nach einem Zusammenschluß, der diese ernsten Zeiten überdauern und in Friedenszeiten durch jährliche Zusammenkünfte die fachliche Fortbildung fördern sollte. Man wollte das «Gesellschaftsspiel» einiger Ordinarii, von dessen Existenz außer mir kaum jemand etwas ahnte, durch eine zeitgemäßere und lebensfähige Arbeitsgemeinschaft ersetzen. Das - zusammen mit einem durch die Dienstleistung gegebenen nationalen Erwachen - bedingte, daß der sich in der alten Richtung bewegende Vorschlag des «Franzo-sen» Hauduroy von den «Militärbakteriologen» geschlossen ab-gelehnt wurde. An dessen Stelle erging eine bereits vorher ge-plante, vom Kurskommandanten gezeichnete, auf den 25. März 1942 lautende Einladung zu einer Gründungsversammlung, wo-bei sich die Liste der Geladenen von Anfang auf die Vertreter der verschiedensten mikrobiologischen Disziplinen erstreckte. Obwohl die Idee bei Welsch- und Deutschschweizern auf lebhafte Sym-pathie stieß, war das Grüpplein, das sich abends 20.00 in einem Sit-zungszimmer des verdunkelten Bahnhofbuffets Zurich einfand, be-scheiden. Die Inhaber der Hygienelehrstühle waren, soweit es Schweizer waren, skeptisch, die Ausländer aus verschiedenen Gründen kraß ablehnend. Ich hatte als Kurskommandant die Sit-zung zu eröffnen - trat dann aber das Präsidium an den statutengewandten Appointé sanitaire L. Badoux ab. In knapp 2 Stunden

10 Jahre Schweizerische Mikrobiologische Gesellschaft

waren die Gesellschaft gegründet und die vorbereiteten Statuten genehmigt. Der Initiative Hauduroys haben wir - nicht zum min-desten als Ausdruck unserer Sympathien für das geplagte Frank-reich - dadu $\Gamma$ ch Rechnung getragen, daß wir ihm noch am sel-ben Abend telegraphisch das Präsidium der 1. Jahresversamm-lung in Lausanne anboten, was er auch annahm, für die Zukunft aber - das soil hier expressis verbis festgehalten werden - der Gesellschaft wenig dankte. 


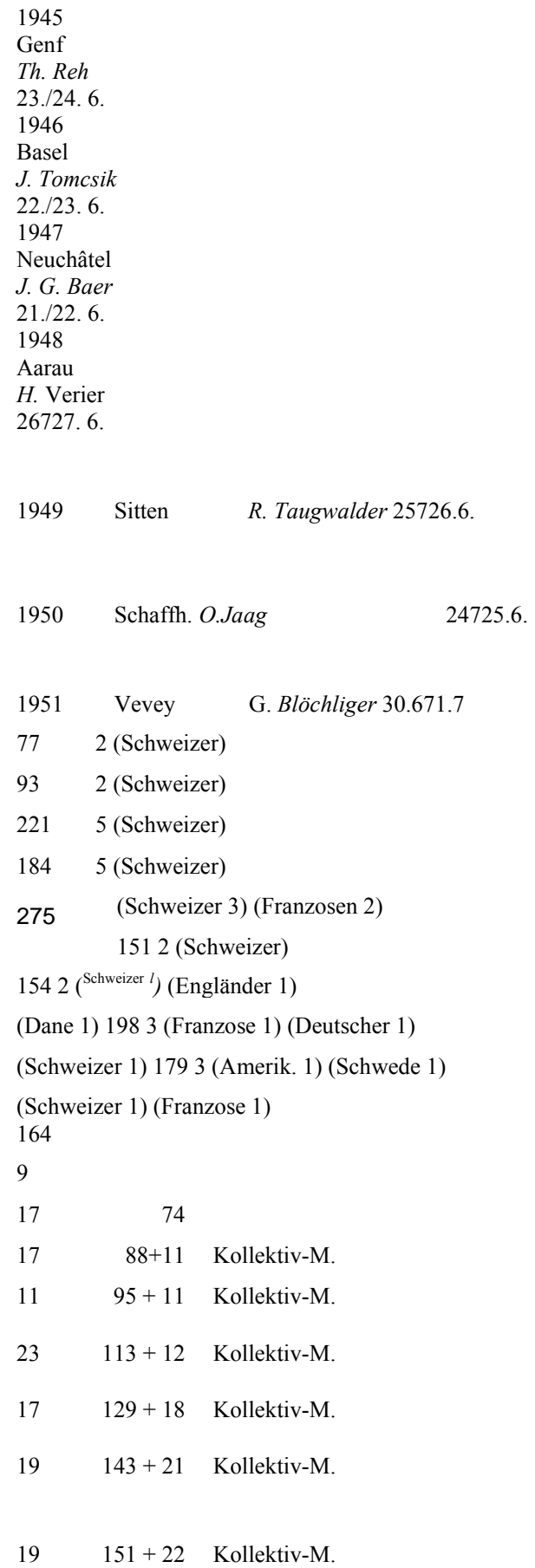

$19168+23$ Kollektiv-M. 
Tabelle I gibt Auskunft über die Entwicklung der Gesellschaft, eine Entwicklung, die in ihrer Konstanz als restlos erfreulich be-zeichnet werden darf. Die Zahl der Mitglieder stieg von 74 im Jahre 1943 (für das Gründungsjahr fehlen mir die Zahlen) auf 172 im Jahre 1951. Darin sehe ich den Beweis dafür, daß die Gründung der Gesellschaft angezeigt und die Konzeption, sie über den medizinischen Rahmen, der ihr zu Gevatter stand, hinauszu-heben und zur Plattform aller an mikrobiologischer Forschung

$642 \quad 10$ Jahre Schweizerische Mikrobiologische Gesellschaft

beteiligten Interessenten - Mediziner und Veterinäre, Phytopa-thologen, Zoologen, Chemiker und Fermentforscher - zu machen, richtig war.

Richtig war wohl auch die schon im ersten Statutenentwurf zum Ausdruck gekommene Tendenz, die Jahresversammlungen im Turnus auf die verschiedenen Landesteile zu verlegen. Dabei ergab sich von selbst, daß zunächst die 5 Universitätsstädte besucht würden. Der jedem Teilnehmer unvergeßliche Empfang, den uns Herr Baer mit seiner Fahrt auf dem Neuenburgersee bereitet hatte, überzeugte jeden, daß man für diese Sitzungen nichts mehr als einen Saal, einen Projektionsapparat und einen «wohlwollen-den» Präsidenten benötigt und damit in der Wahl des Tagungsor-tes frei ist. Mit der für 1952 vorgesehenen Sitzung in Lugano freut sich die Gesellschaft, ihre Visitenkarte zum Beginn des 2. Dezen-niums im Kanton Tessin abgeben zu können.

Etwas weniger konsequent \vurde die Forderung eingehalten, den Vorsitz den Vertretern der verschiedenen Disziplinen abwech-selnd zu überlassen. Immerhin gehörten von den Präsidenten der ersten 10 Jahre 5 der medizinischen und 5 der philosophischen Fakultät II an. Dieser Turnus muß zu Nutz und Frommen der Gesellschaft nicht nur beibehalten, sondern erweitert werden.

Als sehr erfreulich darf der Besuch der Jahresversammlungen bezeichnet werden. Das Datum um den 24./2Õ. Juni kreisend - war offenbar glücklich gewählt und sollte beibehalten werden. Nicht minder erfreulich ist auch das wissenschaftliche Ergebnis dieser Sitzungen. Die Gesellschaft hörte im Laufe der vergange-nen 10 Jahre 31 Referate und Korreferate an. Gemessen am Besuch, darf die Wahl der Themen wie Referenten als im allgemeinen glücklich bezeichnet werden.

Ohne nationalistischen Tendenzen zu frönen, wollten und wol-len wir eine schweizerische Gesellschaft sein. Daß uns das gelun-gen ist, ergibt sich aus der Tatsache, daß 22 von 31 Referaten und Korreferaten durch Schweizer bzw. in der Schweiz ansässige Do-zenten, 4 durch Franzosen und je eines durch einen Amerikaner, Dänen, Deutschen, Engländer und Schweden erstattet wurde, Selbst wenn man berücksichtigt, daß wir die ersten Jahre zwangs-läufig ganz auf uns angewiesen waren, scheint mir das ein Ver-hältnis zu sein, das auch für die Zukunft aufrechterhalten werden sollte.

10 Jahre Schweizerische Mikrobiologische Gesellschaft

TABELLE II.

Themen, über die in den Jahren 1942-1951 Referate und Korreferate etstattet wurden.

1942 H. Mooser (Zurich)

G.Schmid (Bern)

1943 I. Steinmann (Genf) E.Frauchiget (Langenthal)

1944 P. Karrer (Zurich) W. H. Schopfer (Bern)

E. Gãumann (Zurich) J. G. Baer (Neuchâtel) C. Hallauer (Bern)

1945 J. Tomcsik (Basel)

M. Armangué (Genf) 
O. Jaag (Zurich)

O. Morgentha!er (Bern)

R. Regameg (Bern)

1946 R. Meier (Basel)

L.Ettlinger (Zurich)

M. A. Machebceuf (Paris) A. Boivin (Paris) E. Beiger (Basel)

1947 P. Karrer (Zurich) H. Bloch (Basel)

1948 G. Pontecorvo (Glasgow) E. Had orn (Zurich)

1949 K. A. Jensen (Kopenhagen) C. Levaditi (Paris)

G. Domagk (Wuppertal-Elberfeld)

1950 T. Wikén (Zurich)

F. J. Rgn (New York)

Les méthodes actuelles d'immunisation contre le typhus exanthématique.

Die bakteriologische Fleischuntersuchung.

L'état actuel du problème du bactériophage.

Über epideraische Kinderlähmung und ihre Erforschungsmöglichkeiten am Haustier.

Vitamine als Wuchsstoffe für Mikro-organismen.

Immunität und Immunitätsreaktionen bei Pflanzen, Avertebraten und Säugetieren.

Heterogenetische Antigene im allgemeinen. Epiphytismus, Parasitismus und Symbiose. Antibiotica.

Immunochemie.

Über Aminosäuren-decarboxylasen.

Enzymatische Vorgänge bei der Infektion.

Genetics of Micro-organisms.

Beziehungen zwischen allgemeíner Genetik und Genetik der Mikroorganismen.

Humane und bovine Formen der Tuberkel-bazillen.

Etude expérimentale de Гactivité curative antituberculeuse des Antibiotiques d'origine fungique ou végétale.

Die Chemotherapie der Tuberkulose unter Ausschluß der Antibiotica.

Stoffwechselmechanismen bei Gärungs-organismen.

Signification des mutants pour Гétude du métabolisme de fermentation.

B44 10 Jahre Schweizerische Mikrobiologische Gesellschaft

R. Nilsson (Uppsala) Über die intermediären Prozesse des Kohle-

hydratumsatzes in lebender Hefe und in zellfreien Zymasesystemen.

1951 F. E. Lehmann (Bern) Der Kernapparat tierischer Zellen und seine

Erforschung mit Hilfe von Antimitotica.

R. Tulasne (Strasbourg) Quelques notions récentes su la cytologie

des bactéries normales et des bacteries sou-mises à Faction de la pénicilline.

$\mathrm{Zu}$ etwelchen Bedenken gibt dagegen die Zahl der Referate, die an den einzelnen Sitzungen erstattet wurden, Anlaß. Ich daГf das urn so eher sagen, als die Kritik auf mich zurückfällt. Solange wir mit $l 1 / 8$ Tagen, d. h., praktisch gesehen, einem Sitzungstag (Sams-tagnachnrittag und Sonntagvormittag) auskommen sollen, scheint es angezeigt, nicht mehr als 2 Referate, oder 1 
Referat und 1 Korre-ferat ins Programm aufzunehmen. Dadurch gewinnen wir Zeit für Kurzvorträge und die immer und überall zu kurz kommende Diskussion. Gerade hierin möchte ich aber einen wesentlichen Teil der Aufgabe der Gesellschaft erblicken. Tabelle I zeigt, daß die Zahl dieser Kurzvorträge recht konstant ist; sie zu steigern, mögen sich die kommenden Vorstände zur Aufgabe machen. Das gehört m. E. mit zu den Bestrebungen der Förderung des akade-mischen Nachwuchses.

Einen - ich darf sagen, den einzigen - unerquicklichen Punkt in der Geschichte unserer Gesellschaft bildete während langer Jahre die Diskussion um die Publikation der Sitzungsberichte. 1938 haben A. von Albertíní, A. Grumbach und H. Mooser beim Verlag S. Karger A. G., Basel, die Schweizerische Zeitschrift für Allge-meine Pathologie und Bakteriologie gegründet. Damit wollten wir einerseits uns selbst und die Autoren, die es wünschten - und es war eine ansehnliche Zahl der verschiedensten Lander -, vom nationalsozialistischen deutschen Schrifttum unabhängig machen und andererseits auf Schweizer Boden etwas schaffen, das im deutschen und französischen Sprachgebiet verlorengegangen war: die Synthese von allgemeiner Pathologie und Mikrobiologie. Es war naheliegend, die Sitzungsberichte der Gesellschaft dieser Zeitschrift einzuverleiben. Einzelne Mitglieder schienen jedoch zunächst anderer Auffassung zu sein und die diesbezüglichen Diskus-sionen oft recht sehr «zeitgebunden». Immerhin wurde uns zunächst «faute de mieux» der Druck des ersten Sitzungsberichtes überlas-sen; in den nächsten Jahren «pour le mérite» auch der folgenden, 10 Jahre Schweizerische Mikrobiologische Gesellschaft 645

und 1945 erklärte die Gesellschaft die Zeitschrift zu ihrem offiziel-len Organ.

Damit war aber weder die Finanzierung des Sitzungsberichtes noch die der Sonderdrucke, die an die Mitglieder - die von Be-ginn bis heute Fr. 10.- Jahresbeitrag bezahlen - gewährleistet. Wir mußten uns vielmehr, trotz einem dankbar anzuerkennenden Entgegenkommen seitens des Verlages, das Geld von Jahr zu Jahr mühsam zusammenbetteln.

Unter dem Druck der Verhältnisse begann ich anläßlich der ZÜГcher Tagung (ich hatte für die Drucklegung dieses Berichtes Fr. 4000.- aufzubringen) Kollektivmitglieder zu werben. Bereits 1944 war es gelungen, 11 Firmen für die Ziele unserer Gesellschaft zu interessieren. Heute zählt die Gesellschaft 25 Kollektivmitglieder, dank deren Jahresbeiträge es im Jubiläumsjahr mög-lich geworden ist, mit dem Verlag einen Vertrag abzuschließen, der den gegenseitigen Ansprüchen für die nächste Zeit gerecht werden dÜГfte. Gerade deshalb scheint mir dies auch der Zeit-punkt zu sein, wo die Gesellschaft alien ihren z. T. großherzigen Spendern ihren Dank abzustatten hat. Sie haben es ermöglicht, jedem Gesellschaftsmitglied für einen Jahresbeitrag von nur Fr. 10.jedes Jahr die Sitzungsberichte - und es sind dies, wie aus Tab. I hervorgeht, z. T. umfangreiche Bücher - im Sonder-druck zu überlassen.

Ich betrachtete es auch als eine der Aufgaben dieses histori-schen Bückblicks, den Gönnern unserer Gesellschaft durch die in den beiden Tabellen niedergelegten Daten zu zeigen, daß ihre Gründung einem wirklichen Bedürfnis entsprach. Ihre Präsi-denten und Vorstände haben es verstanden, die in unserem Land tätigen Mikrobiologen zu einem international repräsen-tativen Organ zusammenzufassen und damit einem zwar seit Jahr-zehnten gepflegten, aber auf die Zeitschriften unserer Nachbar-länder verzettelten Zweig der Naturwissenschaften schweizerisches Gepräge zu geben. Die Gesellschaft hat einen Sitz im Internationa-len Komitee zur Vorbereitung der Internationalen Kongresse und zwei Sitze im Nomenklaturkomitee der Internationalen Mikro-biologengesellschaft. Sie hat mir die Ehre erwiesen, mich 1945 als Nachfolger von $R$. Doerr in das erstere zu delegieren. Ich konnte in dieser Funktion zweimal, in Kopenhagen und Rio de Janeiro, feststellen, wie gerne es von einer großen Zahl der Delegierten ge-sehen worden ware, wenn sich die Schweiz. Mikrobiologen-Gesell-

$64610 \mathrm{Jah}$ Гe Schweizerische Mikrobiologische Gesellschaft 
schaft bzw. die Schweiz fur die Durchführung eines Inter-nationalen Mikrobiologen-Kongresses zur Verfügung gestellt hätte. Aus äußern Gründen war es mir nicht möglich, den jeweiligen Präsidenten diese Einladung zu überbringen. Ich fürchte, wir haben diese Chance für geraume Zeit verpaßt.

Damit tritt die Gesellschaft in ihr 11. Lebensjahr ein. Möge ihr auch für die Zukunft die stete und ruhige Entwicklung beschieden sein, die ich für das erste Dezennium schildern durfte. Ich möchte denn auch den Rückblick auf ihre erste Entwicklungsperiode nicht beschließen, ohne all den bisherigen Präsidenten und Vorstands-mitgliedern, aber auch jedem einzelnen Mitglied - ob vortragen-des oder zuhörendes - zu danken für das, was es, jedes an sei-nem Platz, zur Entwicklung der Gesellschaft beigetragen hat.

A. Grumbach.

\title{
Mitglieder-Liste \\ der Schweizerischen Mikrobiologischen Gesellschaft
}

Membres

\author{
de la Société Suisse de Microbiologie
}

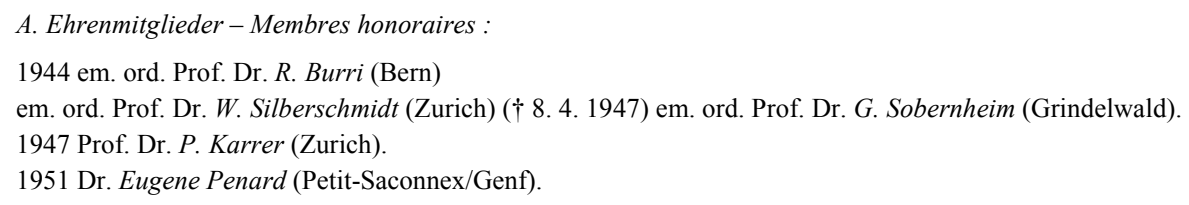

Berenstein, S., Dr., Lausanne, 32, av. de Mon-Repos

Berger, E., P. D. Dr. med., Basel, Pilgerstraße 31

Bernoulli, R., Dr. med., Basel, Rheinsprung 1

Betschard, M., Dr. chem., Brunnen, Lebensmittel-Untersuch.-Anstalt

Bloch, H., P. D. Dr. med., New York, Institute for Public Health, Foot of East

15th Street Blöchliger, G., Dr. ès sc, Gorseaux s. Vevey, Afico S.A. Blumer, S., Dr. phil., Wädenswil, Schönenbergstraße 85 Böhni, E., Frl. Dr., Basel, Falkensteinerstraße 5

Bonifas, V., Dr., Geneve, Inst. d’Hygiène, Laboratoire de bactériologie Bourgeois, Edm., Dr. med. vet., Luzern, Gärtnerstraße 3 Brodhage, H., Dr. med., Davos, Schweiz. Forschungsinstitut Buntzen, T., Dh\, Hindelbank, Hefefabriken A. G. Calpini, P., Chef du Service d'Hygiène de ГEtat du Valais, Sion Castelli, Vero, Dr. ès sc, Lugano, Istituto Biochimico Cathomas, S., Dr. med., Winterthur, Chir. Abt. des Kantonsspitals Chodat, F., Prof. Dr. ès sc, Geneve, Institut de Botanique générale de $\Gamma$ Uni-

veГsité Clerc, Jean-F., Prof. Dr. méd., Neuchâtel, 17, av. de la Gare Cottet, P., Dr. méd., Neuchâtel, Hôpital des Cadolles Dedie, Olivier, Dr. ès sc, Rolle, La Combe Dorner, W., Dr. ès sc, Renens, 2, En Corjon Dufour, L.-F., Lie. ès sc, Geneve, 23, Quai du Mont-Blanc Eckmann, A., Dr. 
chem., Bern, Schweiz. Serum- und Impfinstitut Ettlinger, L., Dr. sc, Institut für spez. Botanik ETH, Zurich Favarger, C, Prof. Dr., Neuchâtel, Institut botanique de ГUniversité Ferrero, A., Dr. ing. chim., Geneve, 1, av. de Miremont Fey, H., Dr., Zurich, Vet.-bakt. Institut der Universität Fischer, H., Prof. Dr., Zurich, Pharmakol. Institut der Universität Fleury, C, Dr. ès sc, Geneve, Institut d'Hygiène, Laboratoire de Bactériologie Flückiger, G., Prof., Eidg. Veterinäramt, Bern Frey, W., Prof., Zurich, Vet.-Pathol. Institut, Manessestraße 4 Fust, Bernh., P. D. Dr. med., Riehen, Fürfelderstraße 26 Gaschen, H. L., Dr. ès sc, Lausanne, 9, Av. W.-Fraisse Gasser, R., Dr. rer. nat., Basel, Kluserstraße 33 Gäumann, E., Prof. Dr., Zurich, Institut für spez. Botanik ETH Geiger-Huber, M., Prof. Dr., Basel, Botanische Anstalt Grasset, E., Prof. Dr., Geneve, Institut d’Hygiène de $\Gamma$ Université Grilichess, R. K., Frl. Dr. med., Zurich, Hygiene-Institut der Universität Gräub, E., Dr. med. vet., Bern, Junkerngasse 41 Gruber, M., dipl. rer. nat., Möhrlistraße 93, Zurich Grumbach, A., Prof. Dr. med., Zurich, Hygiene-Institut der Universität Gsell, O., Dr. med., St. Gallen, Med. Klinik, Kantonsspital Gubler, H. U., Liebefeld-Bern, Eidg. Landw. Versuchsanstalt Haag, E., Dr., Fribourg, Passage du Cardinal Hässig, Alfr., Dr. med., Bern, Schweiz. Rotes Kreuz, Blutspendedienst Hallauer, C, Prof. Dr. med., Bern, Hygiene-Institut der Universität Hauduroy, P., Prof. Dr. méd., Lausanne, Institut d'Hygiène

\section{8}

Mitgliederliste - Membres

Hauser-Hauser, E., FГau Dr., Goßau (Zch.)

Heß, W. R., Prof. Dr., Zurich, Vet.-Bakt. Institut deГ Universität, Selnau-

straße 36 Hirsch, J., Prof. Dr., Basel, J. R. Geigy A.G. Huber, L., Frl., Bern, Schweiz. Serum- und Impfinstitut Hunziker, R. C., Direktor, Rheinfelden, Salmenbräu Hurni, H., Dr., Bern, Landoltstraße 5 Iselin, E., Dr., Basel, Kantonschemiker

Jaag, O., Prof. Dr., Zurich, Institut für spez. Botanik ETH Jadassohn, W., Prof. Dr. méd., Geneve, 38, Av. de Champel Jæger, H., Prof. Dr. méd., Lausanne, 7, Chemin du Levant Kästli, P., Prof. Dr., Liebefeld-Bern, Eidg. Milchwirtschaftl.-Bakt. Anstalt Kent, M., Dr. med., New York, 115 West 73rd Street Kern, H., Dipl. rer. nat., Zurich, Institut für spez. Botanik ETH Klingler, K., Dr., Bern, Vet.-Bakt. und Parasitolog. Institut Kocher, V., Dr., Basel, St.-Alban-Anlage 65 Kradolfer, F., Dr. med., Basel, Engelgasse 93 Kugler, Alf., Dr., Basel, Sandoz A.G. Lang-Porchet, Mme B., Lausanne, 7, Avant-Poste Läuger, P., Dr. phil., Basel, Pelikanweg 9 Leuthardt, Prof. Dr., Rüschlikon, Burai 6 Liska, Josef, Dr., Praha XII, Srobarová 156 Löffler, H., Dr. med., Bern, Hygiene-Institut Löffler, W., Prof. Dr., Zurich, Medizinische Universitätsklinik Lugon, J., Dr. ès sc, Sion, Laboratoire d'analyses médicales Luisier, M., dipl. ing. agr., Sion

Lüthi, H., Dr. phil., Wädenswil, Eidg. Versuchsanstalt Marti, R., Dr., Zurich, Medizinische Klinik, Kantonsspital Meier, R., Prof. Dr., Basel, Gesellschaft für Chemische Industrie, Ciba Messerli, F., P. D., Dr. méd., Lausanne, Villa Helios, Chem. des Bains Metaxas, M. N., Dr. med., Zurich, Hygiene-Institut de $\Gamma$ Universität Mohler, H., Prof. Dr., Zurich, Ausstellungsstraße 90 Moosbrugger, G., Dr., Basel, Eidg. Vakzine-Institut Mooser, H., Prof. Dr. med., Zurich, Hygiene-Institut der Universität Morgenthaler, O., Dr., Bern-Liebefeld, Eidg. Versuchsanstalt Müller, H., Dr. med., Heiden (App.), Kurhaus Quisisana Müller, Th., Dr., Gesundheitsamt des Kts. Basel-Stadt, St.-Alban-Vorstadt 25 Muster, Irene, Dr. ès sc, Geneve, 29, Bd Georges-Favon Naef-Roth, St., Frau Dr., Zurich, Lindenstraße 41 Neipp, L., Dr. ès sc, Basel, Ciba-Aktiengesellschaft Neyroud, M., Dr., Geneve, 1, Rond-Point de Plainpalais Nicole, Alfr., Dr. ès sc, Geneve, 9, rue Cavour Novel, H.E., Dr. ès sc, Geneve, Institut d'Hygiène Payot, F., Dr. méd., Lausanne-Pully, 16, Chamblandes-dessus Peiser, P., Dr., Basel, Alemannengasse 88 Peneveyre, M., Neuilly (Paris), 82 , Charles-Lafitte Plattner, P., Prof. Dr., Zurich, Chem. Institut ETH, Pilliod, G., Dr. ès sc, Montreux, 14, rue du Theatre Mitgliederliste - Membres

\section{9}

Posternak, J., Dr. méd., Lausanne, Institut de Physiologie de ГUniversité Posternak, Mme Y., Lausanne, 10, rue Edouard-Payot Privat, E., Dr. ès sc, Geneve, Labor, d'analyses medicales, 9, rue d'ltalie Radvila, P., Dr. med. vet., Bern, Schweiz. Serum- und Impfinstitut Rambert, J., Ing., Bern, Schweiz. Serum- und Impfinstitut Regamey, R. H., P. D. Dr. med., Bern, Schweiz. Serum- und Impfinstitut Reh, Th., Prof. Dr. méd., Geneve, 10, rue de Hollande Reichstein, T., Prof. Dr., Basel, Pharmazeutische Anstalt Richard, O., Dr. sc. techn., Zurich, Landw. Bakt. Institut ETH Riniker, P., Dr. med., Muralto-Locarno, Le Palme Ritter, P., Ing. agr., Liebefeld-Bern, Eidg. Landw. Versuchsanstalt Ritter, W., Dr. phil., Liebefeld-Bern, Eidg. Milchwirtsch.-Bakt. Anstalt Roch, R., Dr. méd., Geneve, 6, rue Micheli-du-Crest Roggo, J., Dr. ès sc, Fribourg, Institut d'Hygiène Rosenberg, W., Dr., Basel, St.-Alban-Rheinweg 174, Robapharm Rosset, W., Dr. ès sc, Lausanne, 4, Chemin de Chandieu Roth, W., Dr. med., Basel, Hygiene-Institut der Universität Roulet, M. A., Bern, Viktoriastraße 59 Ruzicka, L., Prof. Dr., Zurich, Chem. Institut ETH Sansonnens, R., Dr. med., Bern, Schweiz. Serum- und Impfinstitut Saxer, E., P. D., Dr. med. vet., Gümligen, Weiherstraße 10 Schloßberger, H., Prof. Dr. med., Frankfurt a. M., Hygiene-Institut Schmid, G., Prof. Dr. med. vet., Bern, Vet.-Bakt. Institut 
Schmid, H., Prof. Dr., Zurich, Chem. Institut der Universität

Schneiter, P., P. D., Dr. méd., Lausanne, 14, Av. Tissot

Schnorf, C, Dr. med. vet., Zurich, Veterinaria A.G.

Schoch, A., P. D., Dr. med., Bern, Zeitglockenlaube 2

Schopfer, W., Prof. Dr., Bern, Botanisches Institut der Universität

Schuler, W., Prof. Dr., Arlesheim, Finkelerweg 18

Schwander, L. A., ing. agr., Vevey, Afico S.A.

Schweizer, R., Dr. méd. vét., Lausanne, Institut vétérinaire Galli-Valerio

Seemüller, H., Dr. med. vet., Bern, Schweiz. Serum- und Impfinstitut

Seidenberg, S., Dr. med., Basel, Hygiene-Anstalt

Sinden, J. W., Prof. Dr., Pennsylvania State College, Dept. of Botany, State

College, Pa. USA. Somogy, J. S., Dr., Rüschlikon, Nidelbadstraße 82 Staub, H., Prof. Dr., Basel, Medizinische Universitätsklinik Staub, M., Dr. phil., Zurich, Fehrenstraße 15, Kantonschemiker Steck, W., Prof. Dr., Bern, Veterinärmedizinische Klinik Steinmann, J., P. D., Dr. méd., Geneve, 9, rue de Candolle Stoll, A., Prof. Dr., Basel, Sandoz A.G.

Stoll, Ch., cand. rer. nat., Zurich, Institut für spez. Botanik ETH Suter, E., Dr. med., New York, Rockefeller Institute Taterka, W., Dr. med., Zofingen, A.G. vorm. B. Siegfried Taugwalder, R., Dr. ès sc, Sion, Pharmacie de la Poste Terrier, Ch., Dr. ès sc, Lausanne, 4, Chemin de Rovéréaz Thomann, Dr. O., Zollikon, Dufourstraße 26 Tomarkin, P., Dr. med., Zurich, Seefeldstraße 198

650

Mitgliederliste - Membres

Tomcsik, J., Prof. Dr., Basel, Hygiene-Institut der Universität

Tuescher, J.-L., Dr. ès sc, Leysin, La Gentiane

Ungar, F., Dr. med., Basel, Wettsteinallee 55

Vetter, H., Dr. med., Aarau, Path.-Bakt. Institut des Kantonsspitals

Viscontini, M., Prof. Dr., Zurich, Chemisches Institut der Universität

Vogt, R., Dr., Liebefeld-Bern, Feldrainstraße 48

Vonarburg, H., Tierarzt, Bern, Gutenbergstraße 35

Wälchli, O., Dr., St. Gallen, Dufourstraße 151

Wanner, H., Prof. Dr., Zurich, Institut für allg. Botanik der Universität

Wantz, M., Dr. ès sc, Bern, Schweiz. Serum- und Impfinstitut

Wiesmann, E., Dr. med., St. Gallen, Bakt. Institut

Wikén, T., Prof. Dr., Zurich, Bakt. Institut ETH

Wildhaber, A., Neuchâtel, Bel-Air 1

Wildhaber, M., Dr., Neuchâtel, 2, rue du Peyrou

Wuhrmann, K., Dr., Zurich, Eidg. Anst. f. Wasserversorgung u. Gewässerschutz

Wyttenbach, H., Dr., Basel, Gellertpark 10

Zbinden, Chr., Dr., Vevey, Afico S.A.

Zogg, H., P. D., Dr., Zürich-Oerlikon, Eidg. Landw. Versuchsanstalt

Zollikofer, Ed., Prof. Dr., Zurich, Milchw. Institut ETH

Zurukzoglu, St., P. D., Dr. med., Bern, Wernerstraße 6

C. Kollektivmitglieder-Membres collectifs:

Aktiengesellschaft vorm. B. Siegfried, Zofingen

Bally Schuhfabrik A.G., Schönenwerd

Bell A.G., Basel

Berneralpen-Milchgesellschaft, Stalden-Konolfingen

Cellulose-Fabrik A.G., Attisholz

Elf a, Chemische Fabrik A.G., Aarau

Schweiz. Ferment A.G., Basel

Galactina und Biomalz A.G., Belp (Bern)

J. R. Geigy A.G., Basel

Ed. Geistlich Söhne A.G., Wolhusen (Luzern)

Gesellschaft für Chemische Industrie, CIBA, Basel

Haco A.G., Gümligen

Hero, Konserven A.G., Lenzburg

Hoffmann-La Roche \& Co. A.G., Basel

Schweiz. Käseunion, Bern, Monbijoustraße 47

Laboratoires Om S.A., Geneve, 29, rue des Gares

Maggi-Unternehmungen A.G., Kemptthal 
Sandoz A.G., Basel

Schweiz. Serum- und Impfinstitut, Bern

Société des Produits Nestle S.A., Vevey

Tabacs réunis, Serrières (Neuch.)

Versuchsstation Schweiz. Brauereien, Zurich, Steinhaldenstraße 45

Dr. A. Wander A.G., Bern

Zahnärztliches Institut der Universität Basel

Zyma S.A., Nyon 